\title{
Applying Rational Envelope curves for skinning purposes*
}

\author{
Kinga KRUPPA ${ }^{1,2}$ \\ ${ }^{1}$ Faculty of Informatics, University of Debrecen, Debrecen H-4028, Hungary \\ ${ }^{2}$ Doctoral School of Informatics, University of Debrecen, Debrecen H-4028, Hungary \\ E-mail: kruppa.kinga@inf.unideb.hu
}

Received July 25, 2019; Revision accepted June 27, 2020; Crosschecked Sept. 16, 2020; Published online Nov. 28, 2020

\begin{abstract}
Special curves in the Minkowski space such as Minkowski Pythagorean hodograph curves play an important role in computer-aided geometric design, and their usages are thoroughly studied in recent years. Bizzarri et al. (2016) introduced the class of Rational Envelope (RE) curves, and an interpolation method for $G^{1}$ Hermite data was presented, where the resulting RE curve yielded a rational boundary for the represented domain. We now propose a new application area for RE curves: skinning of a discrete set of input circles. We show that if we do not choose the Hermite data correctly for interpolation, then the resulting RE curves are not suitable for skinning. We introduce a novel approach so that the obtained envelope curves touch each circle at previously defined points of contact. Thus, we overcome those problematic scenarios in which the location of touching points would not be appropriate for skinning purposes. A significant advantage of our proposed method lies in the efficiency of trimming offsets of boundaries, which is highly beneficial in computer numerical control machining.
\end{abstract}

Key words: Medial axis transform; Envelope; Interpolation; Skinning; Circle https://doi.org/10.1631/FITEE.1900377

CLC number: TP391

\section{Introduction}

Medial axis transform (MAT) has been thoroughly studied in both computer graphics and image processing. For a given planar domain, the medial axis (MA) is the locus of centers of maximal inscribed disks, and the MAT can be obtained by lifting the MA to the space using the radii of the inscribed disks. Once we obtain the MAT, we can reconstruct the boundary of the domain using the well-known envelope formula introduced by Choi et al. (1997, 1999). Curves in the Minkowski space are suitable for describing MATs; however, only some special types of curves describe domains whose boundaries are rational. Moon (1999) showed that Minkowski

\footnotetext{
* This work was supported by the construction EFOP-3.6.3VEKOP-16-2017-00002. The project was supported by the European Union, co-financed by the European Social Fund. Open access funding was provided by University of Debrecen (D) ORCID: Kinga KRUPPA, https://orcid.org/0000-0001-53592829

(C) The Author(s) 2020
}

Pythagorean hodograph (MPH) curves are such, and their yielded envelopes are Pythagorean hodograph curves; thus, the envelopes and their offsets are rational as well.

Nowadays, several Hermite interpolation methods have been proposed using MPH curves so that the resulting envelope is rational (Kim and Ahn, 2003; Kosinka and Jüttler, 2006, 2009; Kosinka and Š́r, 2010; Kosinka and Lávička, 2011; Bizzarri et al., 2019). Bizzarri et al. (2016) showed that a broader class of curves exists in $\mathbb{R}^{2,1}$, which yields rational boundaries: the so-called Rational Envelope (RE) curves. Thus, if only the rationality of the envelope is required, one can rely on RE curves, which are easy to compute, whereas one has to restrict oneself to MPH curves only if the rationality of offsets is also needed. The authors described a $G^{1}$ interpolation method to construct an RE interpolant that can be used to create rational blending surfaces between canal surfaces. 
Now we introduce a quite different area in computer-aided geometric design (CAGD) called skinning. Standard point-based curve and surface modeling has always played an important role in CAGD, but there has been a growing interest in modeling based on different objects such as circles and spheres. Skinning has become known as the method of constructing a pair of at least $G^{1}$ continuous splines for a predefined sequence of circles that touches each circle at one point. The idea can also be extended to three-dimensional (3D) modeling, leading to the skinning of an input set of spheres. In recent years, several skinning methods have been developed by Kunkli and Hoffmann (2010), Bana et al. (2014), Bastl et al. (2015), and Kruppa et al. (2019). In addition, skinning can be applied in various fields like computer animation (e.g., ZSpheres ${ }^{\circledR}$ (Pixologic Inc., 2020) and Spore ${ }^{\mathrm{TM}}$ (Electronic Arts Inc., 2008)), molecular biology, and medical image processing (Rossignac et al., 2007; Slabaugh et al., 2008, 2010; Piskin et al., 2017).

Skinning and boundary reconstruction are similar; however, their problem settings are fundamentally different. In skinning, the reconstruction of a boundary is not possible because there is no initial domain assumed at all. Despite this fact, on the basis of the similarities between the two, we propose to approach skinning from the aspect of MAT and use yielded envelope curves.

\section{Motivation and related study}

Let us first consider a parametric curve $\overline{\boldsymbol{y}}(t)=$ $(\boldsymbol{y}(t), r(t))$ as the MAT of a domain. Then the boundary (envelope) of the domain can be reconstructed using the envelope formula (Choi et al., 1997, 1999):

$$
=\boldsymbol{y}(t)-r(t) \frac{r^{\prime}(t) \boldsymbol{y}^{\prime}(t) \pm \boldsymbol{y}^{\prime \perp}(t) \sqrt{\left\|\boldsymbol{y}^{\prime}(t)\right\|^{2}-r^{\prime}(t)^{2}}}{\left\|\boldsymbol{y}^{\prime}(t)\right\|^{2}} .
$$

Next, let us overview the $G^{1}$ Hermite interpolation method proposed by Bizzarri et al. (2016) because the RE curve construction method will be used for our new skinning approach. In Section 2.2, we examine the standard skinning method of Kunkli and Hoffmann (2010).

\section{1 $G^{1}$ Hermite interpolation method yield- ing rational envelopes}

Given two points and two vectors as the input Hermite data for interpolation, let $\boldsymbol{P}_{0}$ and $\boldsymbol{P}_{1}$ denote the endpoints, and $\boldsymbol{t}_{0}$ and $\boldsymbol{t}_{1}$ tangent vectors. Mark projection as $\stackrel{\nabla}{\boldsymbol{n}}=\left(n_{x}, n_{y}\right)$ if $\boldsymbol{n}=\left(n_{x}, n_{y}, n_{z}\right)$ and $\boldsymbol{n} \in \mathbb{R}^{2,1}$. Mark rotation as $\boldsymbol{n}^{\perp}=\left(n_{y},-n_{x}\right)$ if $\boldsymbol{n}=$ $\left(n_{x}, n_{y}\right)$ and $\boldsymbol{n} \in \mathbb{R}^{2}$.

Directly applying the envelope formula (Eq. (1)), we can obtain the touching points $\boldsymbol{Q}_{i}^{ \pm}$ $(i \in\{0,1\})$ of the corresponding envelope curve as follows:

$$
\boldsymbol{Q}_{i}^{ \pm}=\stackrel{\nabla}{\boldsymbol{P}_{i}}-P_{i_{z}} \frac{t_{i_{z}} \boldsymbol{\boldsymbol { t }}_{i} \pm \stackrel{\nabla}{\boldsymbol{t}_{i}^{\perp}} \sqrt{\left\|\boldsymbol{t}_{i}^{\nabla}\right\|^{2}-t_{i_{z}}^{2}}}{\left\|\nabla_{i}\right\|^{2}}
$$

The construction relies on only one branch, and we define the touching point $\boldsymbol{Q}_{i}$ as $\boldsymbol{Q}_{i}^{+}$. We then define appropriate tangent vectors:

$$
\boldsymbol{v}_{i}=\alpha_{i} \frac{\left(\boldsymbol{Q}_{i}-\stackrel{\nabla}{\boldsymbol{P}}_{i}^{\perp}\right.}{\left\|\boldsymbol{Q}_{i}-\stackrel{\nabla}{\boldsymbol{P}}_{i}\right\|}, \alpha_{i} \in \mathbb{R} .
$$

Once we obtain $\boldsymbol{Q}_{i}$ and $\boldsymbol{v}_{i}$, construct the planar curve $\boldsymbol{x}(t)(t \in[0,1])$ that interpolates $\boldsymbol{Q}_{i}$ and $\boldsymbol{v}_{i}$, for example, by Hermite interpolation.

The final step is to construct the MA $\boldsymbol{y}(t)$ as a one-sided offset of $\boldsymbol{x}(t)$ with varying distance:

$$
\boldsymbol{y}(t)=\boldsymbol{x}(t)+r(t) \frac{\boldsymbol{x}^{\prime \perp}(t)}{\left\|\boldsymbol{x}^{\prime}(t)\right\|}=\boldsymbol{x}(t)+f(t) \boldsymbol{x}^{\prime \perp}(t),
$$

which is rational only if $f(t)=r(t) /\left\|\boldsymbol{x}^{\prime}(t)\right\|$ is a rational function. To assure that the resulting curve interpolates the initial input data $\boldsymbol{P}_{i}$ and $\boldsymbol{t}_{i}$, $f(t):[0,1] \rightarrow \mathbb{R}$ must be constructed as a polynomial function with the following boundary conditions $(i \in\{0,1\})$ :

$$
\left\{\begin{aligned}
& f(i)=\frac{P_{i_{z}}}{\left\|\boldsymbol{v}_{i}\right\|}, \\
& f^{\prime}(i)=-\frac{\boldsymbol{t}_{i} \cdot\left(f(i) \boldsymbol{x}^{\prime \prime}(i)-\boldsymbol{v}_{i}^{\perp}\right)}{\nabla} . \\
& \boldsymbol{t}_{i} \cdot \boldsymbol{v}_{i}
\end{aligned}\right.
$$

By lifting the MA back to the space, the final form of the interpolant is as follows:

$$
\begin{aligned}
\overline{\boldsymbol{y}}(t)= & {\left[x_{x}(t)+x_{y}^{\prime}(t) f(t), x_{y}(t)-x_{x}^{\prime}(t) f(t),\right.} \\
& \left.f(t) \sqrt{x_{x}^{\prime 2}(t)+x_{y}^{\prime 2}(t)}\right] .
\end{aligned}
$$


The resulting $\overline{\boldsymbol{y}}(t)$ is a so-called RE curve, a curve yielding a rational envelope. For the construction, refer to Fig. 1. Bizzarri et al. (2016) proved that the envelope is indeed rational. Moreover, if $\boldsymbol{x}(t)$ is a Pythagorean hodograph (PH) curve, then $\overline{\boldsymbol{y}}(t)$ is an MPH curve, and the offsets of the envelope are rational as well.

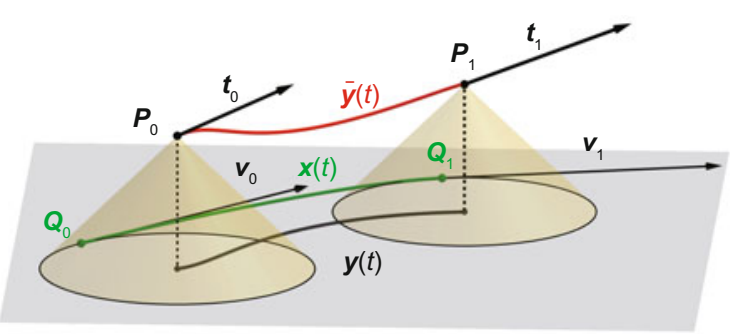

Fig. 1 Construction of the Rational Envelope (RE) curve $\bar{y}(t)$, where $G^{1}$ interpolates the input data $P_{i}$ and $t_{i}(i \in\{0,1\})$ using the method of Bizzarri et al. (2016)

\subsection{Skinning of circles}

As mentioned in Section 1, skinning is a technique used in CAGD for modeling. We introduce the skinning method of Kunkli and Hoffmann (2010). Given an ordered set of circles, skinning is the construction of two $G^{1}$ continuous curves touching each of the given circles at one point separately. One of the most important steps is the localization of the touching points (Fig. 2). The touching points for the first and last circles are determined by the two common outer tangents of the circles; for the other circles, circle triplets are considered. For each triplet, we find two special solutions to the Apollonius problem, using which we can define the touching points for the middle circle. Once we determine points of contact, they are separated into two groups for "left" and "right" skins. Finally, Hermite interpolation curves are constructed between every two neighboring circles for which the tangent lengths are computed using the radical lines of the circle pairs. The resulting $G^{1}$ continuous splines are called skins of the circle set.

The algorithm in Kunkli and Hoffmann (2010) generally provides good results for various input sets, and the skins respond dynamically when the user modifies the positions or the radii of the circles during modeling. Because the touching points are derived using specific solutions to the Apollonius prob- lem, it is also guaranteed that they never lie inside any of the neighboring circles (Fig. 3).

\section{Applying Minkowski Pythagorean hodograph/Rational Envelope curves for skinning purposes}

In this section, we propose a new application area for $\mathrm{MPH} / \mathrm{RE}$ curves: skinning discrete sets of circles. As the problem setting of skinning involves finding bounding curves for an admissible set of predefined circles, we can define the appropriate input set as mentioned in Kunkli and Hoffmann (2010).

Definition 1 Given the ordered set of circles $\mathcal{C}=\left\{c_{1}, c_{2}, \ldots, c_{n}\right\} \quad(n \in \mathbb{N})$ and the corresponding disks $\mathcal{D}=\left\{d_{1}, d_{2}, \ldots, d_{n}\right\}, \mathcal{C}$ is an admissible configuration for skinning if it satisfies the following conditions (Fig. 4):

$$
\begin{aligned}
& \text { 1. } d_{i} \not \subset \bigcup_{j=1, j \neq i}^{n} d_{j}, i \in\{1,2, \ldots, n\} ; \\
& \text { 2. } d_{i} \cap d_{j}=\varnothing, \quad i, \quad j \in\{1,2, \ldots, n\}, j \notin
\end{aligned}
$$

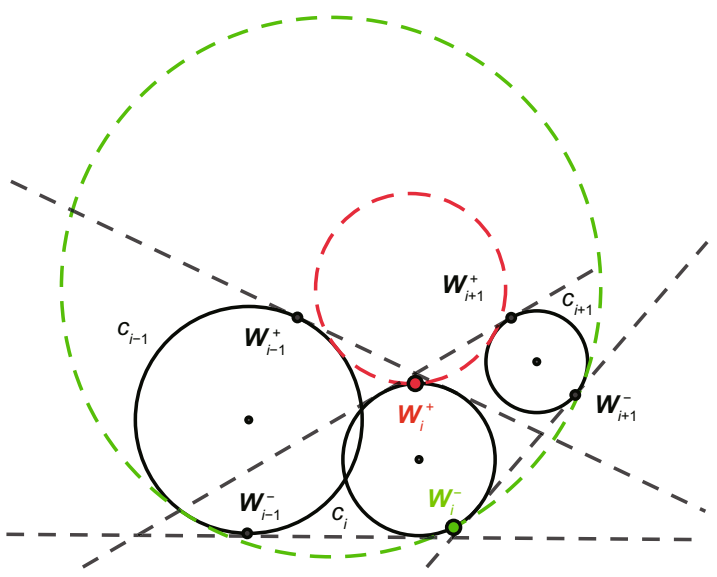

Fig. 2 Determining touching points by the method of Kunkli and Hoffmann (2010) for a sequence of circles: for each circle $c_{i}$, the touching points are determined using specific solutions to the Apollonius problem (red and green); in the case of the first and last circles, the common outer tangents are used. References to color refer to the online version of this figure

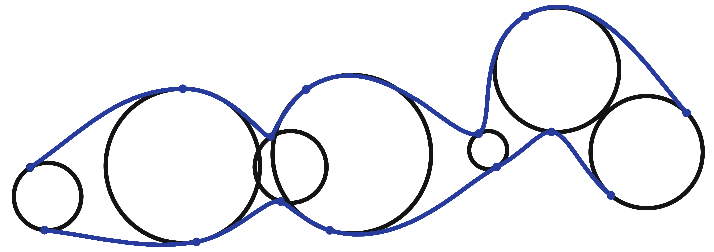

Fig. 3 Skinning circles with the method given by Kunkli and Hoffmann (2010) 
$\{i-2, i-1, i, i+1, i+2\}$

3. $d_{i-1} \cap d_{i+1} \neq \varnothing \Longrightarrow d_{i-1} \cap d_{i+1} \subset d_{i}$, $i \in\{2,3, \ldots, n-1\}$.

To attain an envelope for the admissible sequence of circles $\mathcal{C}$, we need to construct input data for the interpolation. Let us use cyclographic mapping so that we can map the circles to points in $\mathbb{R}^{2,1}$. Thus, we obtain the sequence of points $\mathcal{P}=\left\{\boldsymbol{P}_{1}, \boldsymbol{P}_{2}, \ldots, \boldsymbol{P}_{n}\right\}$, where for each circle $c_{i}$ with center $\boldsymbol{O}_{i}\left(O_{i_{x}}, O_{i_{y}}\right)$ and radius $r_{i}$, the corresponding point $\boldsymbol{P}_{i} \in \mathbb{R}^{2,1}$ is as follows:

$$
\boldsymbol{P}_{i}=\left(O_{i_{x}}, O_{i_{y}}, r_{i}\right) .
$$

The next step is to define $\mathcal{T}=\left\{\boldsymbol{t}_{1}, \boldsymbol{t}_{2}, \ldots, \boldsymbol{t}_{n}\right\}$, the sequence of tangent vectors. However, because we do not have any additional information about the circles, there are no initial tangent vectors provided. We may define the vectors using, e.g., the CatmullRom spline interpolation, so that each $\boldsymbol{t}_{i} \in \mathbb{R}^{2,1}$ tangent vector is defined as $\boldsymbol{t}_{i}=\lambda \cdot\left(\boldsymbol{P}_{i+1}-\boldsymbol{P}_{i-1}\right)$ $(i \in[2, n-1], \lambda \in \mathbb{R} \backslash\{0\})$. For the first and last circles, the vectors are defined as $\boldsymbol{t}_{1}=\lambda \cdot\left(\boldsymbol{P}_{2}-\boldsymbol{P}_{1}\right)$ and $\boldsymbol{t}_{n}=\lambda \cdot\left(\boldsymbol{P}_{n}-\boldsymbol{P}_{n-1}\right)$. Furthermore, using Eq. (2) we calculate positions of the touching points, and after constructing $\overline{\boldsymbol{y}}$ (the RE curve), we obtain the envelope. Generally, this provides adequate output; however, in the case of intersecting circles, the resulting touching point can get inside the neighboring circle, and the envelope can cut into the circles (see an example in Fig. 5). In addition, for $\mathrm{MPH} / \mathrm{RE}$ curves, tangent vectors must be space-like (i.e., their angle with the $x$ - $y$ plane must be less than $45^{\circ}$ ). Taking all these factors into consideration, we cannot use RE curves for skinning by arbitrarily choosing tangent vectors.

To solve this problem, we can approach it from an inverse aspect. In Section 2, we have discussed that the method of Kunkli and Hoffmann (2010) localizes the touching points by solving the Apollonius problem; thus, it guarantees that the points never lie inside any of the neighboring circles. Our idea is to localize the touching points at first and reconstruct precisely that tangent vector with which we would obtain these touching points.

\subsection{Reconstructing tangent vectors}

For each circle $c_{i}$ in $\mathcal{C}$, fix two points that we want to use later as the touching points of the envelope. Mark the left point as $\boldsymbol{W}_{i}^{+}$and right one as

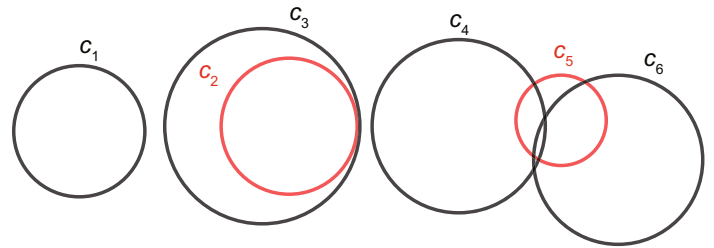

Fig. 4 An example of nonadmissible configuration: by changing, e.g., the positions of the red circles according to Definition 1, we can define both "left" and "right" touching points on every circle. References to color refer to the online version of this figure

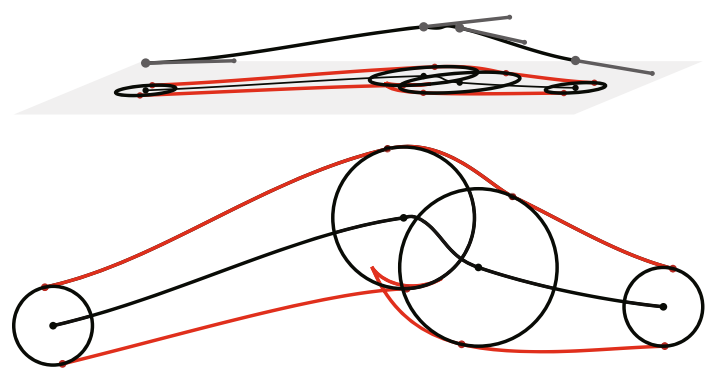

Fig. 5 Using seemingly appropriate tangent vectors that are chosen freely, we observe that the touching points may lie inside the circles

$\boldsymbol{W}_{i}^{-}$. The aim is to determine the tangent vector for which we obtain $\boldsymbol{W}_{i}^{ \pm}=\boldsymbol{Q}_{i}^{ \pm}$using Eq. (2).

It is known that the envelope formula describes a geometric construction (Pottmann and Peternell, 1998; Peternell et al., 2008). Kunkli (2009) also presented different ways to obtain touching points for skinning, one of which was using the geometrical approach to create the corresponding touching points. We use this approach as an inspiration to reconstruct the tangent vector $\boldsymbol{t}_{i}$ from the touching points.

Let $\boldsymbol{W}_{i}^{+}$and $\boldsymbol{W}_{i}^{-}$denote the left and right touching points on $c_{i}$, respectively (Fig. 2). We aim to construct $\boldsymbol{S}_{i}$, the endpoint of the desired tangent vector. Define line $e_{i}=\overleftrightarrow{\boldsymbol{W}_{i}^{+} \boldsymbol{W}_{i}^{-}}$and line $f_{i}$ so that $\stackrel{\nabla}{\boldsymbol{P}_{i}} \in f_{i}$ and $f_{i} \perp e_{i}$. Let $\boldsymbol{I}_{i}$ denote the intersection point of $e_{i}$ and $f_{i}$. $\quad \boldsymbol{S}_{i}$ can now be defined as the inverse point of $\boldsymbol{I}_{i}$ with respect to circle $c_{i}$.

$$
\begin{gathered}
S_{i_{x}}=P_{i_{x}}+r_{i}^{2} \frac{W_{i_{y}}^{+}-W_{i_{y}}^{-}}{\gamma_{i}}, \\
S_{i_{y}}=P_{i_{y}}-r_{i}^{2} \frac{W_{i_{x}}^{+}-W_{i_{x}}^{-}}{\gamma_{i}}, \\
\gamma_{i}=P_{i_{x}}\left(W_{i_{y}}^{-}-W_{i_{y}}^{+}\right)+P_{i_{y}}\left(W_{i_{x}}^{+}-W_{i_{x}}^{-}\right) \\
+W_{i_{y}}^{+} W_{i_{x}}^{-}-W_{i_{x}}^{+} W_{i_{y}}^{-} .
\end{gathered}
$$

Once the endpoint $\boldsymbol{S}_{i}$ is obtained, the tangent 
vector $\boldsymbol{t}_{i} \in \mathbb{R}^{2,1}$ can be practically defined as follows:

$$
\boldsymbol{t}_{i}=\left[\frac{r_{i}^{2}}{\gamma_{i}}\left(W_{i_{y}}^{+}-W_{i_{y}}^{-}\right), \frac{r_{i}^{2}}{\gamma_{i}}\left(W_{i_{x}}^{-}-W_{i_{x}}^{+}\right),-r_{i}\right] .
$$

Geometric construction of $\boldsymbol{t}_{i}$ is shown in Fig. 6 . Because our construction is exactly the inverse of the standard geometric construction of the envelope points, it is trivial that once we define $\boldsymbol{t}_{i}$ this way, the points $\boldsymbol{Q}_{i}^{+}$and $\boldsymbol{Q}_{i}^{-}$obtained using Eq. (2) are the same as $\boldsymbol{W}_{i}^{+}$and $\boldsymbol{W}_{i}^{-}$, respectively. Besides, choosing the tangent vector this way assures that $\boldsymbol{t}_{i}$ is always space-like.

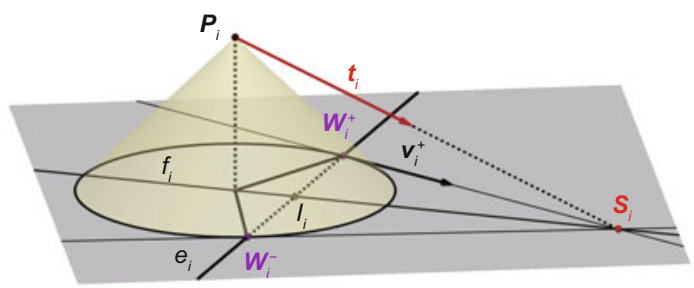

Fig. 6 Reconstructing tangent vector $t_{i}$ using fixed touching points $W_{i}^{+}$and $W_{i}^{-}$. The construction is based on the geometric properties of the envelope of a family of circles

\subsection{Constructing envelope curves}

We have seen that the RE method defined by Bizzarri et al. (2016) uses the $\alpha_{i}$ values in Eq. (3) as free parameters, which essentially affect the shape of the resulting envelope, as $\overline{\boldsymbol{y}}(t)$ is constructed from the planar curve $\boldsymbol{x}(t)$. Because we now use the touching points mentioned in Kunkli and Hoffmann (2010), it is natural to choose the tangent length that the authors suggest. Let $l_{i}$ denote the radical line of $c_{i}$ and $c_{i+1}$. To assure a more aesthetically satisfying result, we differ the starting and ending tangent lengths for each neighboring circle pair. Thus, for the circle pair $c_{i}$ and $c_{i+1}$, the corresponding tangent vectors $\boldsymbol{v}_{i}$ and $\boldsymbol{v}_{i+1}$ for the touching point are defined as follows:

$$
\boldsymbol{v}_{j}=\alpha_{j} \frac{\left(\boldsymbol{W}_{j}^{+}-\stackrel{\nabla}{P}_{j}\right)^{\perp}}{\left\|\boldsymbol{W}_{j}^{+}-\stackrel{\nabla}{\boldsymbol{P}}_{j}\right\|}, j \in\{i, i+1\} .
$$

The $\alpha_{j}$ values can be determined using the Euclidean distance between $\boldsymbol{W}_{j}^{+}$and $l_{i}$ :

$$
\alpha_{j}=2 \operatorname{dist}\left(\boldsymbol{W}_{j}^{+}, l_{i}\right) .
$$

To define the MA, we now need only to follow the remaining steps of the algorithm in Bizzarri et al. (2016). After defining $\boldsymbol{x}(t)$ as a rational planar curve interpolating $\boldsymbol{W}_{i}^{+}, \boldsymbol{W}_{i+1}^{+}, \boldsymbol{v}_{i}$, and $\boldsymbol{v}_{i+1}$ (e.g., as a Hermite arc), we construct the polynomial function $f$ with the boundary conditions (Eq. (4)), and obtain the final RE curve in the form of Eq. (5). The resulting $\overline{\boldsymbol{y}}$ is an RE curve, for which by applying Eq. (1), the resulting envelope curves are rational.

Fig. 7 shows our solution so that the resulting envelope curves are always adequate for skinning. Bizzarri et al. (2016) proved that if we construct $\boldsymbol{x}(t)$ as a PH curve, not only the envelope curves but also their offsets are rational. Fig. 8 shows such a scenario in which the resulting offsets are rational too.

\section{Discussion}

By applying our new algorithm for more complex systems of circles, we can create aesthetic

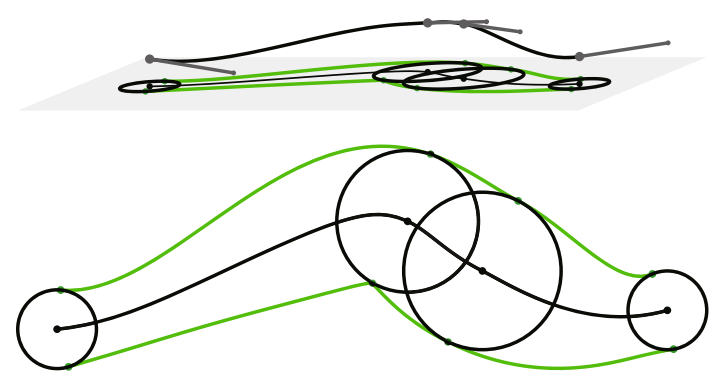

Fig. 7 For the same input as in Fig. 5, we observe that the touching points now lie outside the neighboring circles; thus, if we reconstruct the tangent vectors from the previously chosen touching points, we are able to use the RE curve for skinning

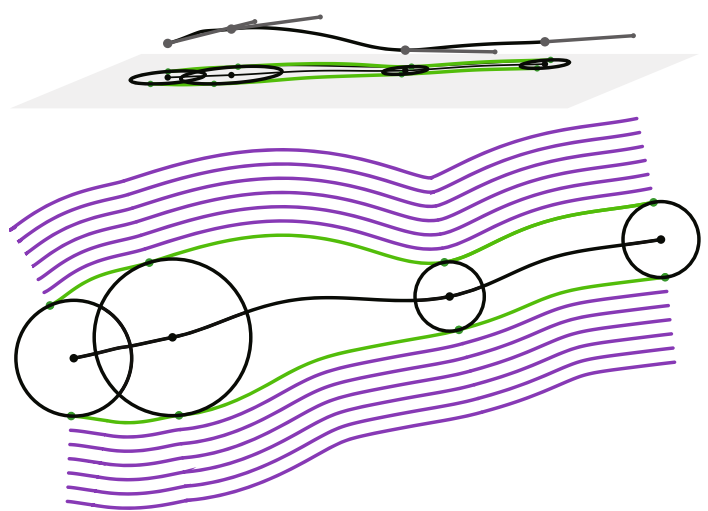

Fig. 8 Minkowski Pythagorean hodograph curve used for skinning with our new approach. Not only the envelope curves but also the offsets are rational 
models. In Fig. 9, we can see various examples: a seahorse (which has been used as a model in existing skinning methods, motivated by Kunkli and Hoffmann (2010)), a snake, and a pair of knife and spoon. We give adequate results for shapes that include many more circles with different radii as well. Because the concept is based on the MAT and thus the input circles behave as maximal inscribed disks of the planar shape, the resulting envelope may have singularities in extreme positions. However, we can simply overcome these situations by adding more circles to describe the desired shape more precisely.

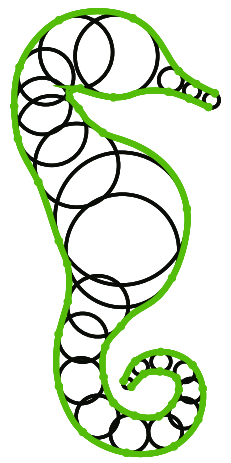

(a)

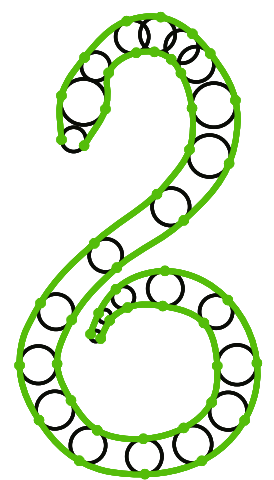

(b)

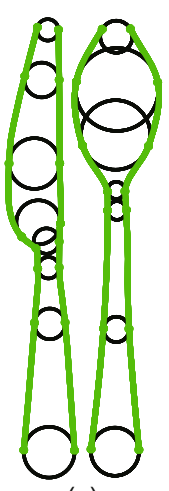

(c)
Fig. 9 Complex and aesthetic two-dimensional models created with our proposed method using the envelopes of RE curves: (a) seahorse; (b) snake; (c) a pair of knife and spoon

The most significant advantage of applying RE and MPH curves for skinning is that they can be used efficiently in computer numerical control (CNC) machining. The detection of self-intersections of the offset curves and the trimming process are fundamental issues, especially in CNC machining and robot path planning, as the intersection of the offsets would cause machine failure in the milling process. Offset curves are trimmed to remove unwanted parts based on their self-intersections, which is a highly expensive and computationally difficult operation for general free-form curves. However, using MATs, we can construct them in an easy and straightforward way (Choi et al., 1999, 2008; Cao and Liu, 2008). For a given $\overline{\boldsymbol{y}}(t)$ (RE/MPH curve), the inner $\delta$-offsets of the envelope curves can be directly constructed as

$$
\boldsymbol{x}_{\delta}^{ \pm}(t)=\boldsymbol{y}(t)+(r(t)-\delta) \boldsymbol{m}^{ \pm}(t),
$$

where $\boldsymbol{m}^{ \pm}(t)$ is obtained by orthogonally projecting the normal vectors of the $\overline{\boldsymbol{y}}$ curve. Trimming the $\delta$-offset curves has now become quite simple: those $\boldsymbol{x}_{\delta}^{ \pm}\left(t_{0}\right)$ parts have to be trimmed where $r\left(t_{0}\right)<\delta$. Thus, if we create circle-based models (such as logos, ornaments, and artistic shapes) by skinning them with $\mathrm{MPH} / \mathrm{RE}$ curves, we assure that $\mathrm{CNC}$ machines can efficiently mill them as we can provide trimmed offsets. An example of the trimming process can be seen in Fig. 10.

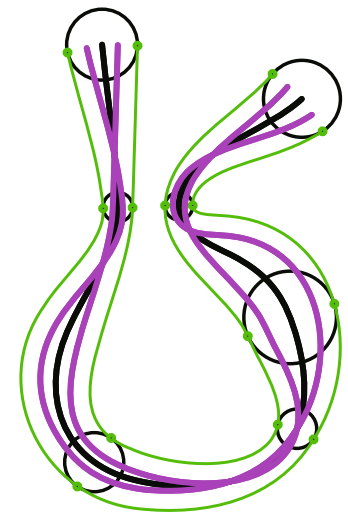

(a)

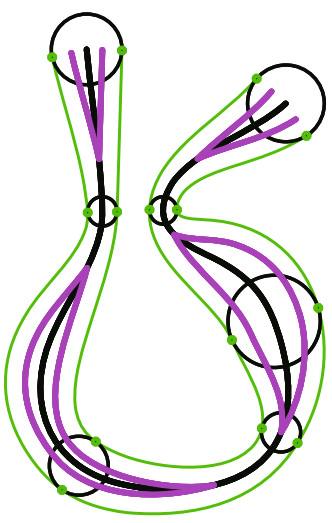

(b)
Fig. 10 Envelope curves with untrimmed (a) and trimmed (b) offsets: using RE curves for skinning purposes, the offset trimming process is efficient and straightforward

\section{Conclusions}

The RE curves were introduced by Bizzarri et al. (2016), and they presented a $G^{1}$ interpolation method to construct an $\mathrm{RE}$ curve in the $\mathbb{R}^{2,1}$ Minkowski space, which yields a rational envelope. Moreover, they can be constructed so that the offsets are rational as well. In this study, we propose a new application for RE/MPH curves: skinning a discrete set of circles. To obtain the envelope, we first need to define the appropriate Hermite input data. We show that circles can be regarded as spatial points using cyclographic mapping, but there is no information about the tangent vectors that should be used for the interpolation. Unquestionably, the shape of the envelope fundamentally depends on the chosen tangent vectors. Even by choosing a seemingly suitable method to define these vectors, the resulting touching points and shape of the envelope are sometimes problematic. To resolve this problem, we offer an inverted approach: we predefine the positions of the touching points (by the 
method of Kunkli and Hoffmann (2010)), and then calculate which is the appropriate tangent vector that would precisely provide the same touching points. We also give an exact calculation to fix the free parameters of the method by Bizzarri et al. (2016), which significantly affect the shape of the envelope as well. Thus, our method does not need a numerical optimization afterward to generate valid output. We give an exact solution, and thus, guarantee that there exists only one envelope for a user-defined circle sequence. In the study, we give all the necessary algorithmic details for the proper construction of the RE curves, for which the envelope curves are adequate for skinning. We also provide several examples of our proposed construction. A significant advantage of this new approach is the efficient offset trimming process, which is fundamentally beneficial, for example, in $\mathrm{CNC}$ machining. For future study, we plan to extend our algorithm to $3 \mathrm{D}$ modeling to provide another application for RE curves: skinning a discrete set of spheres.

\section{Compliance with ethics guidelines}

Kinga KRUPPA declares that she has no conflict of interest.

\section{Open access}

This article is licensed under a Creative Commons Attribution 4.0 International License, which permits use, sharing, adaptation, distribution and reproduction in any medium or format, as long as you give appropriate credit to the original author(s) and the source, provide a link to the Creative Commons licence, and indicate if changes were made. The images or other third party material in this article are included in the article's Creative Commons licence, unless indicated otherwise in a credit line to the material. If material is not included in the article's Creative Commons licence and your intended use is not permitted by statutory regulation or exceeds the permitted use, you will need to obtain permission directly from the copyright holder. To view a copy of this licence, visit http://creativecommons.org/licenses/by/4.0/.

\section{References}

Bana K, Kruppa K, Kunkli R, et al., 2014. KSpheres-an efficient algorithm for joining skinning surfaces. Comput Aided Geom Des, 31(7-8):499-509. https://doi.org/10.1016/j.cagd.2014.08.003

Bastl B, Kosinka J, Lávička M, 2015. Simple and branched skins of systems of circles and convex shapes. Graph
Models, 78:1-9.

https://doi.org/10.1016/j.gmod.2014.12.001

Bizzarri M, Lávička M, Kosinka J, 2016. Medial axis transforms yielding rational envelopes. Comput Aided Geom Des, 46:92-102.

https://doi.org/10.1016/j.cagd.2016.05.006

Bizzarri M, Lávička M, Vršek J, 2019. Linear computational approach to interpolations with polynomial Minkowski Pythagorean hodograph curves. J Comput Appl Math, 361:283-294 https://doi.org/10.1016/j.cam.2019.04.029

Cao LX, Liu J, 2008. Computation of medial axis and offset curves of curved boundaries in planar domain. Comput Aided Des, 40(4):465-475.

https://doi.org/10.1016/j.cad.2008.01.002

Choi HI, Choi SW, Moon HP, 1997. Mathematical theory of medial axis transform. Pac J Math, 181(1):57-88. https://doi.org/10.2140/pjm.1997.181.57

Choi HI, Han CY, Moon HP, et al., 1999. Medial axis transform and offset curves by Minkowski Pythagorean hodograph curves. Comput Aided Des, 31(1):59-72. https://doi.org/10.1016/S0010-4485(98)00080-3

Choi HI, Choi SW, Han CY, et al., 2008. Two-dimensional offsets and medial axis transform. Adv Comput Math, 28(2):171-199. https://doi.org/10.1007/s10444-007-9036-5

Electronic Arts Inc., 2008. Spore ${ }^{\mathrm{TM}}$ and Spore ${ }^{\mathrm{TM}}$ Creature Creator. Redwood City, CA, USA. https://www.spore.com [Accessed on June 17, 2020].

Kim GI, Ahn MH, 2003. $C^{1}$ Hermite interpolation using MPH quartic. Comput Aided Geom Des, 20(7):469492. https://doi.org/10.1016/j.cagd.2003.06.001

Kosinka J, Jüttler B, 2006. $G^{1}$ Hermite interpolation by Minkowski Pythagorean hodograph cubics. Comput Aided Geom Des, 23(5):401-418. https://doi.org/10.1016/j.cagd.2006.01.004

Kosinka J, Jüttler B, 2009. $C^{1}$ Hermite interpolation by Pythagorean hodograph quintics in Minkowski space. Adv Comput Math, 30(2):123-140. https://doi.org/10.1007/s10444-007-9059-y

Kosinka J, Lávička M, 2011. A unified Pythagorean hodograph approach to the medial axis transform and offset approximation. J Comput Appl Math, 235(12):34133424. https://doi.org/10.1016/j.cam.2011.02.001

Kosinka J, Šír Z, 2010. $\quad C^{2}$ Hermite interpolation by Minkowski Pythagorean hodograph curves and medial axis transform approximation. Comput Aided Geom Des, 27(8):631-643. https://doi.org/10.1016/j.cagd.2010.04.005

Kruppa K, Kunkli R, Hoffmann M, 2019. An improved skinning algorithm for circles and spheres providing smooth transitions. Graph Models, 101:27-37. https://doi.org/10.1016/j.gmod.2018.12.001

Kunkli R, 2009. Localization of touching points for interpolation of discrete circles. Ann Math Inform, 36(1):103110.

Kunkli R, Hoffmann M, 2010. Skinning of circles and spheres. Comput Aided Geom Des, 27(8):611-621. https://doi.org/10.1016/j.cagd.2010.07.003

Moon HP, 1999. Minkowski Pythagorean hodographs. Comput Aided Geom Des, 16(8):739-753. https://doi.org/10.1016/S0167-8396(99)00016-3 
Peternell M, Odehnal B, Sampoli ML, 2008. On quadratic two-parameter families of spheres and their envelopes. Comput Aided Geom Des, 25(4-5):342-355. https://doi.org/10.1016/j.cagd.2007.10.007

Piskin S, Unal G, Arnaz A, et al., 2017. Tetralogy of fallot surgical repair: shunt configurations, ductus arteriosus, and the circle of Willis. Cardiovasc Eng Technol, 8(2): 107-119. https://doi.org/10.1007/s13239-017-0302-5

Pixologic Inc., 2020. ZBrush 2020: ZSpheres ${ }^{\circledR}$. Los Angeles, CA, USA.

https://pixologic.com/zbrush/features/ZSpheres/ [Accessed on June 17, 2020].

Pottmann H, Peternell M, 1998. Applications of Laguerre geometry in CAGD. Comput Aided Geom Des, 15(2):165186. https://doi.org/10.1016/S0167-8396(97)00023-X
Rossignac J, Whited B, Slabaugh G, et al., 2007. Pearling: 3 D interactive extraction of tubular structures from volumetric images. MICCAI Workshop on Interaction in Medical Image Analysis and Visualization.

Slabaugh G, Unal G, Fang T, et al., 2008. Variational skinning of an ordered set of discrete 2D balls. Proc $5^{\text {th }}$ Int Conf on Advances in Geometric Modeling and Processing, p.450-461.

Slabaugh G, Whited B, Rossignac J, et al., 2010. 3D ball skinning using PDEs for generation of smooth tubular surfaces. Comput Aided Des, 42(1):18-26. https://doi.org/10.1016/j.cad.2009.03.004 